\title{
FREE BOUNDARIES AND NON-SMOOTH SOLUTIONS TO \\ SOME FIELD EQUATIONS : VARIATIONAL CHARACTERIZATION THROUGH THE TRANSPORT METHOD
}

\author{
J, J. MOREAU \\ Laboratoire de Mécanique Générale des Milieux Continus \\ Université des Sciences et Techniques du Languedoc \\ 34060 MONTPELLIER-Cédex, France
}

\section{INTRODUCTION}

Some methods primarily devised for the optimization of domains are currently used also in the numerical treatment of problems, arising from Physics or Engineering, which involve unknown boundaries. This requires, of course, that the location of the said boundaries could be characterized variationally.

To the author's knowledge, it is in the dynamics of inviscid fluids that variational statements of such a sort have been first proposed. In that field of applications, the unknown surfaces may represent the free boundary through which a liquid confines an atmosphere with negligible inertia and given pressure; they may also describe a jet boundary, in a possibly compressible flow, i.e. a discontinuity locus of the hydrodynamic field, separating two parts of the fluid with preserved material identity (shock waves do not fall into the scope of this lecture). Results in that line have been known since the fifties [1] [2] . In recent papers [3] [4], the author has shown that, when considered from the viewpoint of the dynamics of the whole material in presence, the determination of such surfaces is a problem whose nonlinearity has the same intimate structure as the nonlinearity of the conventional equations of fluid dynamics holding in the regions of smooth flow. This is made clear by expressing dynamics in terms of Schwartz's distributions; 
in doing it, one puts forward the vector distribution divergence of a second order tensor measure associated with the time-space distribution of mass and velocity. For instance, the free boundary of a liquid appears as a surface, interior to the investigated region of time-space, across which the material density abruptly drops to the zero value corresponding to the assumedly mass-less atmosphere. Expressing the dynamics of the whole in terms of distributions encompasses the usual equations, verified in the liquid domain, as well as the conditions to be satisfied on the free surface.

Some details on this aspect of dynamics are given in Sect. 2 below ; for brevity only steady flows are considered.

The next step consists in giving a variational significance to the divergence operator acting on second order tensor measures. When the traditional calculus of variation is applied, with a view to characterize the possible solutions to some field equations, an alteration of the investigated field is performed by adding to it a term, arbitrary in a certain class of functions. This additive variation has to be smooth enough for the familiar trick of integration by part to work ; such a procedure leaves invariant the location of possible singularities and so is unable to characterize it. For this reason, we have instead proposed the transport method (called in [3] and [4] the method of horizontal variations; it seems preferable to abandon this denomination which could generate confusion with some other uses of the word "horizontal", in Differential Geometry).

In this method every alteration of the investigated object is effected by transporting it along an arbitrarily chosen smooth vector field, say $\varphi$, with compact support in the considered region of $\mathbb{R}^{n}$. This vector field may be viewed as the velocity field of some imagined continuous medium $\Lambda$, called a carrier. When the technique is used in a problem of continuum mechanics, one should keep in mind that the carrier has nothing to do with the material in presence ; in particular, the real variable indexing the evolution of $\Lambda$ in $\mathbb{R}^{\mathfrak{n}}$ is denoted by $T$, not to be confused with the time $t$ of Dynamics, when the latter figures among the problem variables. For every position of the carrier, a certain real functional, involving the transported object, is calculated. The result is a real 
function of $\tau$; expressing that its derivative vanishes at $\tau=0$, whatever is the test vector field $\varphi$, yields the expecced characterization. Such a derivation, applied to a geometric object transported along a vector field is known in Differential Geometry as a Lie derivation. For the applications we have in view, it seems more efficient to describe the transport process in terms of the classical kinematics of continua, using but a little of the formalism of Differential Geometry.

Section 3 provides the necessary information about the concepts of vector and tensor distributions on a differential manifold, with emphasis on the special case where distributions are actually measures.

The transport of such objects by what we have called a carrier is described in Section 4.

This yields in Section 5 the very simple formula by which the divergence operation, acting on a doubly contravariant tensor measu$r e$, is interpreted variationally in terms of transport. The real functional to be extremized is the integral of the Euclidean trace of the investigated tensor measure. The reader specially interested in Mechanics could additionally refer to [3], where Hamilton's principle of the least action is connected with this formula.

Section 6 gives some examples of applications of the preceding to hydrodynamical situations.

Then comes in Section 7 the study of the second derivative of the considered real functional, in the course of any twice differentiable transport, if the investigated tensor measure makes the first derivative vanish. This provides a necessary condition for the functional to achieve a local minimum. The calculation results in some positivity property, concerning the investigated tensor measure, which tends to explain the preeminence of measures, in that context, over distributions of higher order.

The final Section 8 summarizes the logical pattern of the transport method and sketches its application to more general situations. 
2. STEADY FLOW OF AN INVISCID FLUID

In some region of a tridimensional reference frame, with orthonormal Cartesian coordinates $x_{1}, x_{2}, x_{3}$, the steady motion of an inviscid fluid is considered. Let $u_{1}, u_{2}, u_{3}$ denote the components of the velocity field; let $p$ and $\rho$ be the pressure and density scalar fields. The components of the gravity field are given, equal to the partial derivatives $U_{, i}$ of some real function $U$. Then, under the usual smoothness assumption for the investigated fields, the Euler time-independent equations of fluid dynamics write down as

$$
\rho u_{j} u_{i, j}=-p, i+\rho u_{, i},
$$

to be joined with the equation of mass conservation

$$
\left(p u_{j}\right)_{j}=0
$$

By combination, this yields $\left(\delta_{i j}\right.$ is the Kronecker symbol)

$$
\left(\rho u_{i} u_{j}+p \delta_{i j, j}\right)_{, i}
$$

The left-hand side may be seen as the i-component of the vector field divergence of the symmetric tensor field with components in brackets.

If on the contrary the involved functions are not smooth enough for the partial derivatives to exist in the elementary sense, it is generally admitted that such partial differential equations as above have to be understood with reference to the partial derivatives of distributions. This is an abuse of language; in Schwartz's theory of distributions, a real function $f$ of the x-variables cannot constitute a distribution. But, as soon as $f$ is locally integrable relative to the Lebesgue measure $\ell$, there is defined the real measure $f \ell$ i.e. the measure admitting $f$ as density relative to $\ell$. A real measure on some region of $x$-space is a special sort of Schwartz distribution; then (2.3) will precisely be replaced by

$$
\left(\rho u_{i} u_{j}^{\ell}+p \delta_{i j}^{\ell)}, j=\rho u, i^{\ell} .\right.
$$


In order that the tensor measure with components in brackets make sense, one supposes $\rho \in L_{\text {loc }}^{\infty}, u_{i} \in L_{1 \text { loc }}^{2}, p \in L_{\text {loc }}^{1}$ (the components $\mathrm{u}, \mathrm{i}$ of the gravity field are essentially smooth functions).

Similarly (2.2) expresses that the vector measure, admitting as Cartesian components the three real measures $\rho u_{i} \ell$, has zero divergence in the sense of distributions.

Before showing how this formulation of hydrodynamics may encompass some free boundary situations, let us recall an elementary calculation rule for distributions.

Assume that the considered domain $\Omega$ of the $x$-space is divided by a surface $S$ determining two subdomains $\Omega^{+}$and $\Omega^{-}$. At every point of $S$, denote by $n_{i}$ the components of the normal unit, directed toward $\Omega^{+}$and assumed continuous on $S$. If a function $f$ is $C^{l}$ in $\Omega^{+}$and $\Omega^{-}$and possesses respective onesided limits $f^{+}$and $f^{-}$at every point of $S$, the measure $f \ell$ is a distribution on $\Omega$ whose partial derivative relative to $x_{i}$ is easily found equal to the sum of the two following measures :

(a) $f, i^{\ell}$, diffuse in $\Omega$.

(b) $\left(f^{+}-f^{-}\right) n_{i} s$ concentrated on $s$.

Here $s$ denotes the area measure of $S$, a nonnegative real measure on $\Omega$, with $S$ as support.

Coming back to hydrodynamics, suppose that $\Omega^{-}$contains an inviscid fluid, while $\Omega^{+}$corresponds to an atmosphere with $\rho=0$. Mass conservation is expressed by $\left(\rho u_{j} \ell\right), j=0$; through the above calculation rule, this is equivalent to (2.2) being satisfied in $\Omega^{+}$and $\Omega^{-}$and to the vanishing of the measure concentrated on $S$ whose density relative to the area measures equals $\left[\left(\rho u_{j}\right)^{+}-\left(\rho u_{j}\right)^{-}\right] n_{j}$. Since $\rho=0$ in $\Omega^{+}$, the latter simply yields $\rho^{-u_{j} n_{j}}=0$, as expected for a steady flow. After that, the same calculation rule is applied to (2.4). Considering, on one hand, the diffuse part, one obtains that (2.3) holds in $\Omega^{-}$ and $\Omega^{+} ;$in particular this yields that $p$ equals a constant throughout $\Omega^{+}$. As for the part of its left-hand member concentrated on $S$, equation (2.4) yields 


$$
\rho^{-} u_{i} u_{j} n_{j}+p^{-} n_{i}=\rho+u_{i}^{+} u_{j}^{+} n_{j}+p^{+} n_{i}
$$

Since $\rho^{+}=0$ and $\rho^{-} u_{j}^{-} n_{j}=0$, this is just the pressure cond $i-$ tion $\mathrm{p}^{+}=\mathrm{p}^{-}$.

3. VECTOR AND TENSOR MEASURES ON A MANIFOLD

A possible way of constructing measure theory consists in taking the Riesz representation theorem as a definition. From that standpoint, by a real (signed) measure on a locally compact topological space $X$ (one may precise : a Radon measure) is meant a real linear functional, meeting some continuity requirements we shall recall later, defined on the space $D^{\circ}(X)$ of the continuous real functions with compact support in $X$. The treatise of Bourbaki [5] is developed in that 1ine, up to include the more sophisticated matter of measures with values in topological linear spaces.

In particular, the considered locally compact space may be an n-dimensional $c^{k}$-differential manifold $M$, with $k \geq 1$. Then the above amounts to define measures as distributions of a special sort. Let $\ell$ be an integer, $0 \leqslant \ell \leqslant k$; denote by $D^{l}(M)$ the Iinear space of the $c^{\ell}$ real functions on $M$ with compact support. For every compact subset $k$ of $M$, denote by $D_{K}^{\ell}(M)$ the subspace of $D^{\ell}(M)$ consisting of the functions $f$ with support contained in $K$. A Banach norm $\|f\|_{K, l}$ is defined on $D_{K}^{\ell}(M)$ as the sup of the absolute values of $f$ and of its partial derivatives up to order $\ell$ at all points of $M$; of course, this is conditioned by the choice of coordinates in the $C^{k}$ manifold $M$, but in view of $\ell \leqslant k$, any admissible change of coordinates (more precisely the change of a covering of $M$ with local charts for another one) replaces the said norm by an equivalent one. By definition, a real distribution of order $\ell$ (strictly speaking, one should say "of order $\leqslant \ell^{\prime \prime}$ ) is a linear functional on $D^{\ell}(M)$ whose restriction to every $D_{\mathrm{K}}^{\ell}$ is continuous.

Real distributions of order $\ell$ on $M$ make a topological linear space denoted by $D^{l}(M)$; real measures on $M$ are the elements of $D^{\circ}(M)$.

In the author's view, the above duality construction serves 
the purpose of Mechanics very well. Generally, dual linear spaces have been a basic ingredient of Classical Mechanics much before the concept was mathematically formalized. This duality is in fact the essence of the method of "virtual power" or "virtual work" which has played a central role in Mechanics since the 17 th century at least (some authors trace it back to Aristotle).

Another definite advantage of this approach of measures is that it readily adapts to the introduction of vector measures on the manifold $M$. With every point $x$ of $M$ is associated the dual pair of n-dimensional linear spaces $M_{x}^{\prime}$, the tangent space, and $M_{x}^{\prime *}$ the cotangent space. By definition a field of covectors on $M$ (or section of the cotangent fiber bunde) is an assignement associating with every $x \in M$ an element, say $v(x)$ of $M_{x}^{1 *}$. Basically, the choice of a (local) coordinate system $\left(x^{1}, x^{2}, \ldots, x^{n}\right)$ in $M$ induces respective bases in the linear spaces $M_{x}^{\prime}$ and $M_{x}^{*}$. Then the covector field $v$ may be described by $n$ functions $v_{i}\left(x^{1}, x^{2}, \ldots, x^{n}\right)$ expressing the components of $v(x)$ in $M_{x}^{*}$. Since, by axiom, any change of (local) coordinate system in $M^{x}$ is $C^{k}, k \geqslant 1$, the concept of the continuity of $v$ is coordinate-free, as well as that of the support of such a field. We shall denote by $D^{\circ}\left(M, M^{1 *}\right)$ the linear space of the continuous covector fields on $M$ with compact support and by $D_{K}^{\circ}\left(M, M^{* *}\right)$ the subspace consisting of those fields whose support is contained in some compact subset $K$ of $M$. The sup of the absolute values of the components of $v \in D_{K}^{\circ}\left(M, M^{* *}\right)$ constitutes a Banach norm $\|\mathrm{v}\|_{K}$ on this space; changing the coordinate system (more precisely changing the covering of $M$ by local charts) replaces this norm by an equivalent one.

By definition, a vector measure on $M$ is a real linear functional on $D^{\circ}\left(M, M^{*}\right)$ whose restriction to each $D_{K}^{0}\left(M, M^{* *}\right)$ is continuous. More generally, such a duality procedure has been used by $G$. de Rham when constructing his theory of currents on $c^{\infty}$ manifolds [9].

Vector measures on $M$ make a topological linear space denoted by $D^{\circ}\left(M, M^{\prime}\right)$. Observe that, in contrast with the special case where $M$ is an open subset of $\mathbb{R}^{\mathrm{n}}$, a vector measure on an arbitrary $C^{k}$ manifold $M$ can by no means be seen as an additive vec- 
tor function of sets since it does not make sense to add vectors localized at different points of the manifold.

When applied to a covector field $v \in D^{\circ}\left(M_{1} M^{* *}\right)$, a vector measure $\mathrm{m} \in \mathcal{D}^{\prime}{ }^{\circ}\left(M, M^{\prime}\right)$ yields, by definition, a real number denoted by «v, m» or, more expressively by $\int\langle v, d m\rangle$. A soon as $m$ is fixed the meaning of this symbol may be extended to a larger class of covector fields than $D^{\circ}\left(M, M^{* *}\right)$, said integrable relative to $\mathrm{m}$. From the Radon-Nikodym theorem one easily deduces that every $m \in D^{\circ}\left(M, M^{\prime}\right)$ can be (non uniquely) represented under the form $\mathrm{m}=\mathrm{m}_{\mu}^{\prime} \mu$ where $\mu$ is a nonnegative real measure on $M$ and $m_{\mu}^{\prime}$ is a locally $\mu$-integrable vector field. This means that for every $v \in D^{\circ}\left(M, M^{*}\right)$, one has

$$
\int\langle\dot{i}, \mathrm{dm}\rangle=\int\left\langle\mathrm{v}(\mathrm{x}), \mathrm{m}_{\mu}^{\prime}(\mathrm{x})\right\rangle \mathrm{d \mu} ;
$$

here $\langle.$, . $\rangle$ denotes, at every point $x$ of $M$, the real-valued duality pairing of the cotangent and tangent spaces.

Incidentally, a vector measure $m \in D^{\circ}\left(M, M^{\prime}\right)$ is said

divergence-free if, for every $\varphi \in D^{1}(M)$, one has

$\int\langle\operatorname{grad} \varphi, \mathrm{dm}\rangle=0$; we denote by $\operatorname{grad} \varphi$ the gradient field of $\varphi$ (or differential of $\varphi$ ), naturally an element of $D^{\circ}(M, M)$.

In [6] this concept has been applied to classical hydrodynamics, yielding a generalization of the Kelvin-Helmholtz theorem on vorticity which encompasses more recent results as the conservation of the helicity of a flow. More generally, the divergence of a vector measure on $M$ may be defined as a scalar distribution of order 1 . The point to be stressed is that this operation makes sense in the simple framework of the differential manifold $M$, without reference to any metric or connection; this contrasts with the divergence of vector fields.

A similar duality device may be generally used in defining tensor distributions, in particular tensor measures, on the $c^{k}$ manifold $M$. For instance, there exist four sorts of second order tensor fields on $M:$ they are assignements associating respectiveIy with every $x \in M$ an element of $M_{x}^{\prime} \otimes M_{x}^{\prime}, M_{x}^{\prime} \otimes M_{x}^{*}, M_{x}^{* *} \otimes M_{x}^{\prime}$, or $M_{x}^{1 *} \otimes M_{x}^{1 *}$. This allows for the definition of spaces $D^{\ell^{x}}, D_{K}^{l}$ consisting of such fields and, through duality, the definition of 
spaces of tensor distributions of order $\&$ with specified tensorial type. In particular, a doubly contravariant tensor measure on $M$ is an element of $D^{\circ 0}\left(M, M^{\prime} \otimes M^{\prime}\right)$, the dual space of $D^{\circ}\left(M, M^{*} \otimes M^{* *}\right)$; the latter consists of continuous doubly covariant tensor fields on $M$, with compact supports. As in the case of vector measures, the Radon-Nikodym theorem may be used in order to prove that, for every $\mathrm{T} \in D^{\circ}\left(M, M^{\prime} \otimes M^{\prime}\right)$, there exist (non uniquely) a nonnegative real measure $\mu$ and a doubly contravariant locally $\mu$-integrable tensor field $\mathrm{T}_{\mu}^{\prime}$ such that $\mathrm{T}=\mathrm{T}_{\mu}^{\prime} \mu$. Through the use of local coordinates $\left(x^{1}, \ldots, x^{n}\right)$ in $x$, one defines the tensor field $x \rightarrow T_{\mu}^{\prime}(x) \in M_{x}^{\prime} \otimes M_{x}^{\prime}$ by its components $T_{\mu}^{, i j}$, which are elements of $L_{\text {loc }}^{\mu}(x, \mu ; \mathbb{R})$; one may even choose $\mu$ in order that they belong to $L_{\text {loc }}^{\infty}(X, \mu ; \mathbb{R})$.

\section{CARRIERS AND LIE DERIVATIVES}

For all the sequel, $X$ denotes a fixed n-dimensional $c^{k}$ manifold, $k \geqslant 2$; in usual applications, $X$ simply reduces to an open subset of $\mathbb{R}^{\mathbf{n}}$.

Let $\varphi \in D^{1}\left(X, X^{\prime}\right)$, i.e. $\varphi$ is a $C^{!}$vector field of $X$, with compact support. We are to look at it as the Eulerian velocity field of some continuum $\Lambda$ in motion over $X$. This precisely means that every element, or particle, of $\Lambda$ is a moving point in $x$, say $\tau \rightarrow \xi(\tau)$ verifying the differential equation

$$
\frac{d \xi}{d \tau}=\varphi(\xi(\tau))
$$

Through the use of (local) coordinates in $x$, the study of this differential equation in the manifold may be reduced to the similar problem in $\mathbb{R}^{n}$, for which standard theory is available. The assumptions made about $\varphi$ secure that, for every $\xi_{0} \in X$, there exists a unique solution $\tau \rightarrow \xi(\tau)$ to (4.1), defined for $\tau$ ranging over the whole real line, such that $\xi(0)=\xi_{0}$. Furthermore, standard results concerning the dependence on initial conditions of the solutions to differential equations, entail that, for every fixed $\tau$, the mapping $\xi_{0} \rightarrow \xi(\tau)$, commonly denoted by $\exp \tau \varphi$, is $\mathcal{C}^{l}$ of $X$ to itself. Since this mapping admits $\exp (-\tau) \varphi$ as inverse, it constitutes a $c^{l}$ diffeomorphism of $X$, 
leaving invariant every point of the subset $X_{\backslash}$ support $\varphi$.

Let us express as $\tau \rightarrow p(\tau, \lambda)$ the motion of a particle $\lambda$ of $\Lambda$ relatively to $X$; under the usual wording of the kinematics of continua, for every $\tau$, the mapping $p_{\tau}: \lambda \rightarrow p(\tau, \lambda)$ is called the placement of $\Lambda$ into $X$ at time $T$. The above statements equivalently mean that, for every $T$, the placement $p_{\tau}: \Lambda \rightarrow X$ is one-to-one and that the "transplacement" $p_{\tau} \circ p_{0}^{-1}$ is a $C^{l}$ diffeomorphism of $X$. We shall in turn reformulate this by saying that the set $A$ may be equipped with the structure of a $C^{l}$ manifold, in such a way that, for every $\tau$, the placement $p_{\tau}$ is a $c^{l}$ diffeomorphism of $\Lambda$ onto $X$.

Commonly in Mechanics, time derivatives are denoted by a dot. In accordance, $\dot{p}(\tau, \lambda)$ will refer here to the derivative of the mapping $\tau \rightarrow p(\tau, \lambda)$ of $\mathbb{R}$ into $X$; for every $\tau$ and $\lambda$, this derivative is an element of the tangent space $x_{x}^{\prime}, x=p(\tau, \lambda)$. The differential equation (4.1), with which all began, manifests itself as the identity

$$
\forall \tau \in \mathbb{R}, \forall \lambda \in \Lambda \quad: \quad \dot{\mathrm{p}}(\tau, \lambda)=\varphi(\mathrm{p}(\tau, \lambda)) \text {. }
$$

We shall call a carrier such moving differential manifold as $\Lambda$, elaborated from a given $C^{l}$ vector field $\varphi$ in $X$. For simplicity, we started with $\varphi$ independent of $\tau$, i.e. the motion of $\Lambda$ over $X$ is a steady flow. It is sometimes useful to consider more generally a vector field $x \rightarrow \varphi(\tau, x)$ in $x$, depending on $\tau$, at least for $\tau$ ranging over some open real interval $I$ containing zero; then $\varphi$ will be supposed $C^{l}$ in $\tau$ and $x$ jointly (equivalently $(\tau, x) \rightarrow(1, \varphi(\tau, x))$ is a $c^{l}$ vector field of the product manifold $I \times X$; ; in addition the support of $x \rightarrow \varphi(\tau, x)$, for every $\tau \in I$, will be assumed contained in a $T$-constant compact subset of $X$. Again this allows one to equip $\Lambda$ with the structure of a $\mathcal{C}^{\mathrm{l}}$ manifold, in such a way that every placement $\lambda \rightarrow p(\tau, \lambda), \quad \tau \in I$, is a $c^{I}$ diffeomorphism of $\Lambda$ onto $x$. With a $C^{\prime}$ manifold, as are $\Lambda$ or $X$ above, one may associate various linear spaces, respectively consisting of real functions, vector or tensor fields, distributions of order $\leqslant 1$, etc. For every element of any of these spaces, there is a natural definition 
of its image under any $c^{l}$ diffeomorphism of the considered manifold onto another one ; this image is an object of the same nature attached to the target manifold. The spaces $0^{\circ}, 0^{l}, D^{\prime}, D^{\prime l}$ introduced in the preceding section generate examples of this. As another instance, consider the linear space $C^{l}(\Lambda, \mathbb{R})$ of the continuously differentiable real functions on the $c^{\prime}$ manifold $\Lambda$ and some $c^{1}$ diffeomorphism $p: \Lambda \rightarrow X$. For every real function $k: \Lambda \rightarrow \mathbb{R}$, the image under $p$ is naturally defined as the function $k=\kappa o p^{-1}: X \rightarrow \mathbb{R}$, which belongs to $C^{l}(X, \mathbb{R})$ iff $\kappa \in C^{l}(\Lambda, \mathbb{R})$. Similarly may be considered a vector field on $\Lambda$, say $\lambda \rightarrow \alpha(\lambda) \in \Lambda_{\lambda}^{\prime}$. Its image under $p$ is defined as $x+p_{\lambda}^{\prime}(\alpha(\lambda))$, with $\lambda=\mathrm{p}^{-1}(\mathrm{x})$ and $\mathrm{p}_{\lambda}^{\prime}: \Lambda_{\lambda}^{\prime} \rightarrow X_{\mathrm{x}}^{\prime}$ the tangent linear mapping to $p$ at point $\lambda$. This image belongs to $C^{\circ}\left(X, X^{\prime}\right)$ iff $\alpha \in C^{\circ}\left(\Lambda, \Lambda^{\prime}\right)$. For a covector field on $\Lambda$, the image is analogously defined, only using instead of $P_{\lambda}^{\prime}$ its inverse transpose.

Let us come back to the kinematical setting; then $D_{\tau}, \tau \in I$ is the $\tau$-depending $c^{1}$ diffeomorphism of $\Lambda$ onto $X$, generated by the given velocity field $\varphi$. Let $\alpha$ be any of the objects we associated above with the $C^{\prime}$ differential structure of $\Lambda$. Assume $\alpha$ independent of $\tau$; then $i$ ts image under $p_{\tau}$, say $a^{\tau}$, is a $\tau$-dependent object of the same nature related to $X$. Genera1Iy a t-varying object of $X$ derived in that way from a $T$-constant object of $\Lambda$ is said convected by the moving continuum, or carrier, $\Lambda$. This belongs to the vocabulary of usual physics : a function $\alpha$ which assigns to every particle of the moving continuum a timeindependent real value is commonly called a convected quantity. When observed from the "reference manifold" $x$, such a function $\alpha$ : $\Lambda \rightarrow \mathbb{R}$ is reflected as $a^{\tau}: X \rightarrow \mathbb{R} ;$ in the familiar case of $c^{l}$ real functions, one elementarily characterizes convection by the transport equation $\partial a^{\tau} / \partial \tau+\left\langle\varphi, g r a d a^{\top}\right\rangle=0$.

Symmetrically, let us consider now a $\tau$-constant object, say $a$, related to $x$. Its image under $\mathrm{p}_{\tau}^{-1}$ is a ${ }_{\tau}$-dependent object of the same nature, related to $\Lambda$, say $a^{\top}$. Recall that the various classes of objects we agreed to consider constitutes each a linear spaces ; there is usually no difficulty in endowing those respective linear spaces with topologies and, due to the assumptions made 
at the start about the vector field $\varphi$, to show that the derivative $\mathrm{d} \alpha \tau / \mathrm{d} \tau$, at $\mathrm{\tau}=0$, exists, an element of the same linear space. In turn, this derivative admits an image under the placement $\mathrm{p}_{0}$; this is an object, related to the $c^{k}$-structure of $x$, of the same nature as a. Traditionnally this object is denoted by $L_{\varphi} a$ and called the Lie derivative of a along $\varphi$.

We finish this section by recalling an example of Lie derivative which plays an essential role in the classical kinematics of continua. As a $\tau$-constant object of the geometry of $X$, let us take a second order doubly covariant symmetric tensor field $g$ assumed to be $C^{l}$ at least. Practically, this will be the tensor field involved in the definition of a Riemannian metric on $X$ (or a pseudo-Riemannian metric, in Relativity theory) through the writing $d^{2}=g_{i j} d x^{i} d x^{j}$. For every placement $P_{T}$ of the moving continuum $\Lambda$, the image of $g$ under $p_{\tau}^{-1}$ is a doubly covariant symmetric tensor field on $\Lambda$ that we shall denote by $\gamma^{\tau}$. Through the writing $d \sigma^{2}=\gamma_{i j}^{\tau} \mathrm{d} \lambda^{i} \mathrm{~d} \lambda^{j}$, there is defined a metric on $\Lambda$, actually the metric induced on $\Lambda$ by its placement at time $\tau$ in the Riemann space $X$. Saying that $\mathrm{do}^{2}$ depends on $\tau$ means that the continuum $\Lambda$ is not expected to move "rigidly". For every particle $\lambda \in \Lambda, \gamma^{\tau}(\lambda)$ is a $\tau-$ dependent element of the symmetrized tensor product $\Lambda^{\prime *} \otimes_{s} \Lambda_{\lambda}^{\prime *}$. Under the smoothness assumptions previously made, one easily finds that the $T$-derivative $\dot{\gamma}^{\tau}$ exists at $\tau=0$; thereby is defined $\dot{\gamma}^{0} \in \mathcal{C}^{\circ}\left(\Lambda, \Lambda^{*} \otimes_{S} \Lambda^{\prime *}\right)$. The image of $\dot{\gamma}^{\circ}$ under the placement $p_{0}$ : $\Lambda \rightarrow X$ constitutes the Lie derivative $L_{\varphi} g$, a $c^{0}$ doubly covariant symmetric tensor field on $X$.

The calculation of $\mathrm{L}_{\varphi} \mathrm{g}$ is performed, under diverse notations, in any textbook on the Mechanics of Continua; we shall come back to this in Section 7 . Most books are restricted to the usual case where $X$ equals an open subset of a Euclidean space; then some orthonormal Cartesian coordinates are used as $x^{i}$. If the (possibly $\tau$-dependent) velocity field $\varphi$ is described by its components $\varphi_{i}\left(x^{1}, \ldots, x^{n}\right)$ relative to this Cartesian frame, the components of the tensor $L_{\varphi} g$ are found equal to $\partial \varphi_{i} / \partial x^{j}+\partial \varphi_{j} / \partial x^{i}$. In this setting of orthonormal Cartesian coordinates, it is usual to define the differential operator def, acting on differentiable vector 
fields, by writing $e=\operatorname{def} \varphi$ for $e_{i j}=\left(\varphi_{i, j}+\varphi_{j, i}\right) / 2$. Then

$$
\mathrm{L}_{\varphi} \mathrm{g}=2 \operatorname{def} \varphi
$$

Due to coordinates being orthonormal, in our Euclidean space, no distinction has to be made here between covariance and contravariance. In the elementary kinematics of continua, $e$ is usually called the spatial strain rate tensor associated with the Eulerian velocity field $\varphi$. In fact, knowing $2 e=L_{\varphi} g$ enables one to calculate what we have denoted above by $\dot{\gamma}^{\tau}$; thereby may be computed the time-rate of change of the length of any "infinitesimal material curve element", as soon as is known the image of this element in $X$ under the placement $p_{\tau}$.

Actually an expression of $L_{\varphi} g$ formally as simple as (4.3) may be written in the general case where $g$ defines a regular pseudo-Riemannian metric on $X$; one finds (cf. [7])

$$
\left(L_{\varphi} g\right)_{i j}=\varphi_{i \mid j}+\varphi_{j \mid i}
$$

where $\varphi_{i}\left(x^{1}, \ldots, x^{n}\right)$ are the covariant components of the possibly t-dependent velocity field $\varphi$ and where $\mathrm{lj}$ refers to the covariant derivative in the $j$ direction, relative to the pseudo-Riemannian connection.

5. THE METRIC TRACE INTEGRAL OF A TENSOR MEASURE

As before, $x$ denotes a $c^{k}$ manifold, $k \geqslant 2$. A doubly covariant symmetric $c^{1}$ tensor field $g$ is supposed given in $x$; it is intended to define a Riemannian metric on $X$ but, at the present stage, we do not need it to satisfy any positivity condition.

One considers a doubly contravariant symmetric tensor measure $T$ in $X$ such that the integral

$$
J=\ll g, T »=\int g_{i j} d T^{i j}
$$

makes sense, $i . e$. the real measure $g_{i j} d T^{i j}$ is bounded. The real number $J$ will be called the metric trace integral of $T$, relative to $g$. This name is suggested by the special case where $X$ 
equals an open subset of some Euclidean space and $g$ the tensor associated with the Euclidean metric; then, if $x^{1}, \ldots, x^{n}$ are orthonormal Cartesian coordinates, one has $g_{i j}=1$ for $i=j$ and zero otherwise, therefore

$$
J=\int g_{i j} d T^{i j}=\int d T^{i i}=\int \text { trace } d T .
$$

In this special situation, no distinction has to be made between upper and lower indices; $\mathrm{T}^{\mathrm{ii}}$ is a scalar measure, easily proved invariant under any orthogonal change of coordinates. The integral $J$ is meaningful provided this measure is bounded.

Let us define a carrier $\Lambda$ by its velocity field $\varphi \in D^{1}\left(X, X^{\prime}\right)$, possibly depending on $\tau \in I$, an open real interval containing zero, in a $\mathrm{C}^{\mathbf{l}}$ way.

Incidentally, the possible lack of boundedness of the real measure $g_{i j} T^{i j}$ comes from $g$ not having a compact support. The essentials of the calculations we shall perform below may be adapted to such a situation by restricting the integral to a compact subset of $X$ containing, for every $\tau \in I$, the support of $\varphi$.

Denote by $T^{\tau}$ the $\tau$-dependent doubly contravariant symmetric tensor measure on $X$, equal to $T$ for $T=0$ and convected by the carrier. According to the system of definitions developed in Section 4 , this means that a doubly contravariant $t$-constant tensor measure $\theta$ on the manifold $\Lambda$ is introduced as the image of $T$ under $\mathrm{p}_{0}^{-1}$; by definition, $\mathrm{T}^{\tau}$ equals for every $\tau$ the inage of $\theta$ under $p_{\tau}$. We are to calculate the t-derivative, at $\tau=0$, of the function $\tau+\mathrm{J}(\tau)$, the metric trace integral of $\mathrm{T}^{\mathrm{T}}$.

As in Section $4, \gamma^{\tau}$ denotes the image of $g$ under $\mathrm{p}_{\tau}^{-1}$. The definitions of images of fields and measures or distributions are precisely devised in order to preserve the various duality pairings ; in particular, for every $\tau \in I$, one has $\left\langle g, T^{\tau} \gg=\left\langle\gamma \gamma^{\tau}, \theta \gg\right.\right.$. We have seen that, under the smoothness assump-

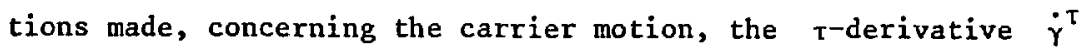
exists, a continuous doubly covariant symmetric tensor field in $\Lambda$ with compact support. Then a standard argument of derivation under the integral symbol (see e.g. [4]) yields 


$$
\frac{d}{d \tau} \varangle g, T^{\top} \gg=2 \ll \operatorname{def} \varphi, T^{\tau} \gg,
$$

a formula to be applied at time $\tau=0$.

In the case where $X$ equals some open subset of an Euclidean space, with orthonormal Cartesian coordinates, no distinction is made between upper and lower indices and, in view of the symmetry of $T$, one has

$$
\begin{aligned}
2 \ll \operatorname{def} \varphi, T^{\tau} \gg & =\ll \varphi_{i, j}+\varphi_{j, i}, T^{i j_{\gg}} \\
& ={ }^{2 \ll \varphi}{ }_{i, j}, T^{i j} j_{\gg} .
\end{aligned}
$$

Now the definition of partial derivatives in the theory of distributions, yields

$$
\ll \varphi_{i, j}, T^{i j} \gg=-\left\langle\varphi_{i}, T^{i j}, j^{\gg}\right.
$$

Therefore the linear functional $\varphi \rightarrow-\varphi_{i, j}, \mathrm{~T}^{i \mathrm{j}_{\gg}}$ is a vector distribution of order one, whose Cartesian component of rank $i$ equals $\mathrm{T}^{\mathrm{ij}}, \mathrm{j}$. This vector distribution will naturally be called the divergence of the tensor measure. $T$, by analogy with the divergence of a tensor field, commonly used in the Mechanics of Continua. In the latter case, $s^{i j}$ denote the components of some $c^{l}$ (symmetric) tensor field, relative to some orthonormal Cartesian frame; partial derivation is understood in the traditional sense, so that the divergence $s^{i j}, j$ is a $c^{\circ}$ vector field. Here is the connection between the two concepts : let $\ell$ denote the Lebesgue measure in our (local1y) Euclidean space $X$; one easily checks that $s^{i j_{\ell}}$ are the components of a tensor measure whose divergence, in distribution sense, equals the vector measure with components $s^{i j}, j^{\ell}$.

As a result of the above calculations, we may formulate the following:

PROPOSITION 5.1 A bounded symmetric tensor measure $\mathrm{T}$ in the 10cally Euclidean manifold $X$ has zero divergence if and only if, for every carrier, as defined in Section 4 , the real function $\tau+\int$ trace $\mathrm{dT}^{\top}$ has zero derivative at $\tau=0 ;$ here $\mathrm{T}^{\boldsymbol{T}}$ denotes 
the doubly contravariant symmetric tensor measure convected by the carrier, equal to $T$ for $\tau=0$.

More generally, (5.2) with (5.3) gives a variational meaning to the divergence operator, acting on symmetric tensor measures. This may be applied in characterizing variationally the solutions to some field equations which assert that such a divergence equals some vector distribution of adequate form. An example of this sort will be presented in the next Section.

of course, it is not necessary to restrict oneself to locally Euclidean manifolds; if $X$ is pseudo-Riemannian everythings works the same way, provided we define the operator

$$
\operatorname{div}: D^{\prime \circ}\left(x, X^{\prime} \otimes_{s} X^{\prime}\right)+D^{\prime 1}\left(x, x^{\prime *}\right)
$$

as the negative transpose of

$$
\operatorname{def}: D^{1}\left(X, x^{\prime}\right) \rightarrow D^{0}\left(x, X^{*} \otimes_{s} x^{*}\right) ;
$$

recall that def denotes the operator $\varphi \rightarrow\left(\mathrm{L}_{\varphi} g\right) / 2$. 


\section{HYDRODYNAMICAL EXAMPLE}

This Section is to show the ability of the transport method to provide a variational treatment of equations in which the divergence of a tensor measure appears jointly with other terms. The setting is that of Section 2 ; we write again equation (2.4) under the form

$$
\left(\rho u_{i} u_{j}^{\ell)}, j+(p \ell), i-\rho U_{, i}^{\ell}=0\right. \text {. }
$$

The tensor measure with components $\rho u_{i} u_{j}{ }^{\ell}$ has a peculiar structure; relatively to the nonnegative real measure $\mathrm{m}=\rho \ell$, it admits as density the tensor field with components $u_{i} u_{j}$, name1y the tensor product of the vector $u$ by itself. In order that such a tensor measure be transported by the carrier $\Lambda$ in the way described in the preceding Section, i.e. that it equal for every $T$ the image under $P_{\tau}$ of some $\tau$-constant doubly contravariant tensor measure on $\Lambda$, it is enough : firstly to have the measure $m$ convected by $\Lambda$, secondly to have the vector field $u$ convected. The former means that a $\tau$-dependent real measure $m^{\top}$ on the space $X$ of the $x$-coordinates, reducing to $m$ for $\tau=0$, equals the image under $p_{\tau}$ of some $\tau$-constant real measure $\mu$ on $\Lambda$. This is the same as the familiar situation of the Mechanics of Continua : then $\mu$ denotes the mass measure, defined independently of time on a material continuum; the image $\mathrm{m}^{\tau}$ of this real measure under the placement mapping $p_{\tau}: \Lambda+X$ admits a density, say $\rho^{\top}$, relatively to the Lebesgue measure $\ell$. Elementarily, the law of transport for $\rho^{\top}$ consists in the following : the value of $\rho^{\top}$ corresponding to any determined particle $\lambda$ of $\Lambda$ verifies $\rho^{\top} J^{\top}=$ const, where $J^{\top}$ denotes the Jacobian determinant of $p^{\top}$ at point $\lambda$ (relative to an arbitrarily chosen coordinate system in $\Lambda$ ).

The metric trace integral of the above tensor measure equals $I(\tau)=\int u_{i}^{\tau} u_{i}^{\tau} \rho^{\tau} d \ell$; that is, formally, twice the kinetic energy of the investigated portion of fluid. As a result of Section 5 , by calculating the $\tau$-derivative at $\tau=0$ of this expression one obtains

$$
\frac{1}{2} \frac{d I}{d \tau}=-\varangle\left(\rho u_{i} u_{j}^{\ell)}, j, \varphi^{i} \gg,\right.
$$


where $\varphi \in D^{l}\left(X, X^{\prime}\right)$ denotes the velocity field of the carrier $\Lambda$. The transport method also gives a variational meaning to the last term in (6.1). Assuming as above that the measure $m^{\tau}=\rho^{\tau} \ell$ is convected by the carrier, one considers the integral $G(\tau)=\int U \rho^{T} d \ell$. In order to calculate its $\tau$-derivative, one uses the fact that $\mathrm{m}^{\tau}$ equals the image of a $\tau$-constant measure on $\Lambda$. When $U$ is evaluated by following up the motion of a particle $\lambda \in \Lambda$ in the reference manifold $X$, one finds

$$
\frac{d}{d \tau} U\left(p_{\tau}(\lambda)\right)=U, i \varphi^{i}
$$

therefore, at $\tau=0$,

$$
\frac{d G}{d t}=\int_{\Lambda} U \frac{d}{d \tau}\left(P_{\tau}(\lambda)\right) d \mu=\int_{X} U \cdot, i^{i} \rho d \ell .
$$

There finally remains to treat the middle term in (6.1). Mechanically, the dynamical equation of inviscid flows have to be exploited in conjunctions with the knowledge of some compressibility law for the investigated fluid. We shall make here the traditional assumption of a barotropic flow, i.e. for every fluid particle, a certain relation between the density $\rho$ and the pressure $p$ is asserted, without explicit recourse to temperature. This holds in particular in the very usual situation where the fluid may be admitted to evolve isentropically. Let us write down this relation as

$$
p=p(k, \sigma)
$$

with $\sigma=\rho^{-1}$ denoting the volume of unit mass. Here $k$ is a variable of arbitrary mathematical nature, assumed to be a constant for every fluid particle; this may refer as suggested above to the entropy of the particle, but, since our main motivation lies in the treatment of sharp inhomogeneities, one has also to be prepared to make $x$ account for the chemical nature, possibly different in various parts of the flow. Traditionally, there is introduced a primitive of $\sigma \rightarrow p(\kappa, \sigma)$, say $P(\kappa, \sigma)$.

We shall apply the transport method to the integral $\int P(\kappa, \sigma) \rho d l$, supposing as before that the measure $m=\rho l$ is convected, i.e. $\mathrm{m}^{\tau}=\rho^{\tau} \ell$ with $\rho^{\tau} J^{\tau}=$ const. for every $\lambda \in \Lambda$. 
On the other hand we agree to effect the transport with $\kappa$ kept independent of $\tau$ for every $\lambda$ and, naturally, with $\sigma$ related to $\rho$ by $\sigma^{\tau}=\left(\rho^{\top}\right)^{-1}$. Under these assumptions one calculates the $\tau$-derivative of $\int \mathrm{P}\left(\kappa, \sigma^{\tau}\right) \rho^{\tau} \mathrm{d} \ell$. By applying to the carrier $\Lambda$ the dilatation formula, classical in the Kinematics of Continua, one has, if $\sigma^{\tau}$ is evaluated for a $\tau$-constant element of $\Lambda$,

$$
\frac{d \sigma^{\tau}}{d \tau}=\sigma^{\tau} \varphi^{i}, i
$$

Then, keeping in mind that $\rho^{\tau} \mathrm{d} l$ equals the image under $P_{\tau}$ of the $\tau$-constant measure $d \mu$ on $\Lambda$, one finds, for $\tau=0$,

$$
\begin{aligned}
\frac{d}{d \tau} \int_{X} P\left(\kappa^{\tau}, \sigma^{\tau}\right) \rho^{\tau} d \ell & =\int_{\Lambda} \frac{\partial P}{\partial \sigma} \frac{d \sigma^{\tau}}{d \tau} d \mu \\
& =\int_{X} p(\kappa, \sigma) \varphi^{i}, i^{\top} \rho^{\tau} d \ell=-\int_{X} P, i^{i} d l
\end{aligned}
$$

since $\varphi$ has compact support.

Adding up the various terms, one obtains (cf. [3], Proposition 10.3):

PROPOSITION 6.1 A vector field $\mathrm{u}$, and three functions $\mathrm{p}, \mathrm{p}, \mathrm{k}$ (with $\sigma=\rho^{-1}$ ) make a solution of (6.1) and (6.4) if and only if, for every carrier with velocity field in $D^{l}\left(X, X^{\prime}\right)$, the $\tau$-derivative of the following functional vanishes at $\tau=0$

$$
B(\tau)=\int\left(\frac{l}{2} u_{i}^{\tau} u_{i}^{\tau}+P\left(\kappa^{\tau}, \sigma^{\tau}\right)+U\right) \rho^{\tau} d \ell ;
$$

here it is assumed that $u^{\top}, \kappa^{\top}, \sigma^{\tau}, \rho^{\top}$, reducing to the above for $\tau=0$, are transported by the carrier in the way defined in the preceding.

Let us finish by stressing that the condition $\left(\rho u_{j} \ell\right), j$ of mass conservation is not involved in the above variational characterization. In that respect, one has to recall the observation made in Section 3 : the concept of a divergence-free vector measure belongs to the geometry of $c^{1}$ manifolds; hence it is preserved under any $\mathcal{C}^{l}$ diffeomorphism such as the placement $p_{T}$ of the carrier $\Lambda$ into $X$. Consequently, for a vector measure convected 
by a carrier, the vanishing of divergence is a $\tau$-invariant property. Now the law of transport defined in the preceding implies that the vector measure with components $\rho u_{j} \ell$ is convected. It will be pointed out in the sequel that the essence of the transport method consists in characterizing some investigated object as a critical point for some real functional in an infinite dimensional manifold. The elements of this manifold result from each other through the transport by carriers. In the present case all of them are divergence-free vector measures ; hence the vanishing of divergence does not constitute a constraint regarding the variational procedures.

An analogous remark applies to the fact that, since the investigated flow is supposed steady, the variable $\kappa$ must assume a constant value along each streamline. This property is evidently preserved when $u^{\tau}$ and $\kappa^{\top}$ are transported by any carrier in the imposed way. 


\section{THE SECOND VARIATION RATE}

Let us come back to the setting of Proposition 5.1. Some symmetric doubly contravariant tensor measure $T$ on the locally Euclidean manifold $X$ is supposed to have zero divergence ; equivalently, in view of the proposition, the trace integral $J(\tau)$ of $T^{\tau}$ has zero time-derivative at $\tau=0$, whatever is the carrier $\Lambda$, with velocity field $\varphi \in D^{l}\left(X, X^{\prime}\right)$.

Incidentally, since the distribution $T^{i j}$ is by assumption a measure, the vanishing of the left-hand member of (5.3) for every $\varphi \in \mathcal{D}^{1}\left(X, X^{x}\right)$ is secured, in view of a density argument, as soon as the same holds for $\varphi$ ranging over the subset $D^{\infty}\left(X, X^{\prime}\right)$, the $C^{\infty}$ vector fields with compact support in $X$.

We are now to investigate the second derivative of the function $\tau \rightarrow J(\tau)$. This of course requires some additional assumption concerning the carrier motion; we shall suppose that $\varphi$ is twice differentiable in $\tau$ and $x$, i.e. the vector field $(1, \varphi)$ of the product manifold $I \times X$ is $C^{2}$.

As before, $\gamma^{\top}$ denotes the image of the tensor field $g$ under $\mathrm{p}_{\tau}^{-1}$. For every $\lambda \in \Lambda$, some element $\dot{\gamma}^{\top}(\lambda)$ of $\Lambda_{\lambda}^{\prime *} \otimes_{s} \Lambda_{\lambda}^{* *}$ constitutes the derivative of $\tau \rightarrow \gamma^{\tau}(\lambda)$ if and only if, for every pair $\alpha, \beta$ of $\tau$-constant elements of $\Lambda_{\lambda}^{\prime}$, one has

$$
\frac{d}{d \tau}\left(\gamma_{i j}^{\tau}(\lambda) \alpha^{i}{ }^{j}\right)=\dot{\gamma}_{i j}^{\tau} \alpha_{\beta}^{i} j
$$

The respective images $a(\tau), b(\tau)$ of $\alpha$ and $\beta$ under $p_{\tau}^{\prime}(\lambda)$ (the tangent linear mapping to $p_{T}$ at point $\lambda$ ) are moving vectors in $X$, associated with the moving point $\tau \rightarrow p_{\tau}(\lambda)$. Since $x$ is locally Euclidean, we shall make use in it of Cartesian coordinates $x^{i}$, nonnecessarily orthonormal. By the definition of $\gamma^{\tau}$, one has, for every $\tau \in I$,

$$
\gamma_{i j}^{\tau}(\lambda) \alpha^{i} \beta^{j}=g_{k l^{a^{k}}(\tau) b^{\ell}(\tau)}
$$

In the terminology of Section 4 , the moving vectors $a(\tau)$ and $b(\tau)$ are convected by the carrier; this is known to be expressed by

$$
\frac{d}{d \tau} a^{k}(\tau)=\varphi^{k}, i(\tau, x) a^{i}(\tau)
$$


with $x=p_{\tau}(\lambda)$, and the similar law for $b(\tau)$ (such a law is nothing but the classical formula concerning the derivation, relative to initial data, for the solutions to the differential equation (4.1)). Since $x^{i}$ are Cartesian coordinates in $x, g$ is a constant, hence the right-hand side of (7.2) possesses the following $\tau$-derivative

$$
\begin{aligned}
& g_{k \ell} \varphi^{k}, i a^{i^{\ell}+g_{k \ell} a^{k} \varphi^{\ell}, j} b^{j} \\
& =\varphi_{\ell, i} a^{i_{b} \ell}+\varphi_{k, j} a^{k} b^{j}=\left(\varphi_{\ell, k}+\varphi_{k, \ell}\right) a^{k} b^{\ell},
\end{aligned}
$$

where $\varphi_{1}, \ldots, \varphi_{\mathrm{n}}$ denote the covariant components of the velocity field $\varphi$. This proves the existence of $\dot{\gamma}^{\tau}$, namely the image, under the tangent mapping to $\mathrm{p}^{-1}$, of the element $2 \mathrm{e}$ of $X_{x}^{\prime *} \otimes_{s} X_{x}^{\prime *}$ with components $\varphi_{\ell, k}+\varphi_{k, \ell}$. What we have done here is only establishing that the tensor field $2 \mathrm{e}$ equals the Lie derivative $\mathrm{L}_{\varphi} \mathrm{g}$, the classical fact from which Proposition 5.1 was deduced. We shall now apply the same technique in calculating the $\tau$-derivative of the expression (7.4). For fixed $\lambda \in \Lambda$,

$$
\begin{aligned}
\frac{d}{d \tau} e_{k \ell}\left(\tau, p_{\tau}(\lambda)\right) & =e_{k \ell, \tau}+e_{k \ell, m} \frac{d}{d \tau} p_{\tau}^{m}(\lambda) \\
& =e_{k \ell, \tau}+e_{k \ell, m^{\varphi}} .
\end{aligned}
$$

Recall that the components of $e$, as well as the components of $\varphi$, essentially are functions of $\tau, x^{1}, \ldots, x^{n} ;$ the notation , $\tau$ refers to the partial derivatives of such functions with respect to $\tau$, evaluated at point $x=p_{\tau}(\lambda)$. Then, using (7.3), one calculates the derivative of $\tau \rightarrow e_{k \ell}\left(\tau, p_{\tau}(\lambda)\right) a^{k}(\tau) b^{\ell}(\tau)$ as follows

$$
\left(e_{k \ell, \tau}+e_{\left.k \ell, m^{\varphi^{m}}\right) a^{k} b^{\ell}+e_{k \ell}\left(\varphi^{k}, i^{a^{i} b^{\ell}}+a^{k} \varphi^{\ell},{ }^{b} j\right.}^{j}\right)
$$

i.e., after renaming some indices of summation,

$$
\frac{d}{d \tau}\left(e_{k l} a^{k} b^{\ell}\right)=h_{k \ell} a^{k} b^{\ell}
$$

with

$$
h_{k \ell}=e_{k \ell, \tau}+e_{k \ell, m} \varphi^{m}+e_{m l} \varphi^{m}, k+e_{k m} \varphi^{m}, l \cdot
$$


Since (7.5) holds whatever is the couple $\alpha, \beta$ of elements of $\Lambda_{\lambda}^{\prime}$ one starts with, this proves the existence of the second derivative $\ddot{\gamma} \tau$ of the function $\tau+\gamma^{\tau}(\lambda)$; it equals the image of the tensor $2 h\left(\tau, p_{\tau}(\lambda)\right)$ under the tangent mapping to $p_{\tau}^{-1}$. We sha11 use this to establish :

PROPOSITION 7.1 Let $T$, a bounded symmetric tensor measure in the locally Euclidean manifold $X$, have zero divergence. Let $\mathrm{T}^{\top}$ denote the doubly contravariant tensor measure convected by the carrier $\Lambda$, equal to $T$ for $\tau=0$. Then the real function $\tau \rightarrow J(\tau)=\int$ trace $\mathrm{dT}^{\tau}$, whose first derivative vanishes at $\tau=0$ in view of Proposition 5.1, admits, as soon as the carrier velocity is $c^{2}$, a second derivative at $\tau=0$ equal to

$$
\ddot{J}(0)=2 \int g_{i j} \varphi^{j}, k^{\varphi^{j}}, \ell^{d T^{k \ell}} \text {. }
$$

Proof. In view of the preceding, $\ddot{\gamma}^{\tau}$ is a continuous doubly covariant tensor field of $\Lambda$, with compact support; $T^{\top}$ equals the image in $X$ of a $\tau$-constant doubly contravariant tensor measure $\theta$ in $\Lambda$. Therefore the standard procedure of derivation under the integral symbol yields

$$
\ddot{J}(\tau)=\int \ddot{\gamma}_{\dot{i} \mathbf{j}} d \theta^{i \mathbf{j}}=2 \int \mathrm{h}_{k \ell} \mathrm{dT}^{\mathrm{k} \ell} .
$$

Due to $T^{k \ell}=T^{\ell k}$, the expression $h_{k \ell}$, symmetric in $k$ and $\ell$, may be replaced by

$$
\begin{aligned}
\varphi_{\mathrm{k}, \ell T} & +\varphi_{\mathrm{k}, \ell \mathrm{m}}{ }^{\mathrm{m}}+\varphi_{\mathrm{k}, \mathrm{m}} \varphi^{\mathrm{m}}, \ell+\varphi_{\mathrm{m}, \mathrm{k}} \varphi^{\mathrm{m}}, \ell \\
& \left.=\varphi_{\mathrm{k}, \tau}+\varphi_{\mathrm{k}, \mathrm{m}} \varphi_{, \ell}^{\mathrm{m}}\right)_{,}+\varphi_{\mathrm{m}, \ell^{\varphi}} \varphi_{\ell}^{\mathrm{m}} .
\end{aligned}
$$

Since $T$ has zero divergence

$$
\int \varphi_{\mathrm{k}, \tau}+\varphi_{\mathrm{k}, \mathrm{ml}}^{\left.\varphi^{\mathrm{m}}\right)}, \mathrm{dT}^{\mathrm{kl}}=0 \quad \text {; }
$$

furthermore $\varphi_{m, k}=g_{m i} \varphi^{i}, k$, so $(7.6)$ is proved.

One may contemplate as follows the use of Proposition 7.1 . Suppose that a doubly contravariant symetric tensor measure, with bounded trace, is given in $X$. The totality of the tensor measures 
which can be obtained from this one through the transport by carriers with $D^{l}$ velocity fields constitutes, roughly speaking, an infinite-dimensional manifold, say M. Proposition 5.1 states that, in this manifold, the elements with zero divergence are the critical points of the trace integral, a real function. Exploring the vicinity of such a critical point through the transport by carriers with $D^{2}$ velocity fields is the object of Proposition 7.1. In particular, the nonnegativity of $\ddot{\vec{J}}$ is a necessary condition for the functional to achieve a local minimum at the considered point. The following Proposition allows one to discuss this condition.

PROPOSITION 7.2 As before, $X$ denotes a locally Euclidean manifold. The expression (7.6) is nonnegative for every $\varphi \in D^{1}\left(X, X^{\prime}\right)$ (equivalently for every $\varphi \in D^{\infty}\left(X, X^{\prime}\right)$ ) if and only if one these equivalent conditions is satisfied :

(a) For every $\psi \in D^{\circ}\left(X, X^{\prime}\right)$ (equivalently for every $\psi \in D^{\infty}\left(X, X^{\prime}\right)$ ) one has

$$
\int \psi_{i} \psi_{j} d x^{i j} \geqslant 0
$$

(b) There exist (non uniquely) a nonnegative scalar measure $\mu$ and functions $\mathrm{T}_{\mu}^{\prime \mathbf{i j}} \in L_{1 \mathrm{oc}}^{\infty}(X, \mu)$ such that $\mathrm{T}^{\mathrm{ij}}=\mathrm{T}_{\mu}^{\prime}{ }^{\mathrm{ij}} \mathrm{j}_{\mu}$ and that for $\mu$-almost every $x \in X$ the quadratic form $\xi \rightarrow \xi_{i}^{\mu} \xi_{j} T_{\mu}^{\prime}{ }^{i j}(x)$ is nonnegative in $\mathbb{R}^{\mathrm{n}}$.

Proof. It is understood that $\psi_{i}$ denote the covariant components of the vector field $\psi$, while the components $T^{i j}$ of the tensor measure are contravariant. Thus, the properties (a) and (b) are invariant under any change of coordinates. Let us take profit of this by assuming that orthonormal Cartesian coordinates are used in $X$; then the expression (7.6) reduces to $2 \int \varphi^{\mathrm{i}}, \mathrm{k}^{\varphi^{\mathrm{i}}}, \ell^{\mathrm{dT}}{ }^{\mathrm{k} \ell}$.

Suppose this expression is nonnegative for every $\varphi \in D^{\infty}\left(X, X^{\prime}\right)$; in particular, one may fix $i_{o} \in\{1, \ldots, n\}$ and assume $\varphi^{i}=\theta \in D^{\infty}(X, \mathbb{R})$ for $i=i_{0}$ and $\varphi^{i}=0$ otherwise. Then the quadratic functional defined on $D^{\infty}\left(X, X^{\prime}\right)$ as $\psi+\int \psi_{k} \psi_{\ell} \mathrm{dT}^{\mathrm{k} \ell}$ is nonnegative for every vector field $\psi$ which equals the gradient of 
some $\theta \in D^{\infty}(X, \mathbb{R})$. Through the use of standard test functions (see e.g. [8]) this may be proved to imply that the quadratic functional is nonnegative for every $\psi \in D^{\infty}\left(X, X^{\prime}\right)$ and therefore, by density, for every $\psi \in D^{0}\left(X, X^{\prime}\right)$; this is property (a). Conversely, the special form that expression (7.6) takes on in orthonormal coordinates makes this expression visibly nonnegative as soon as (a) holds.

Trivially (b) implies (a) . Conversely, we have observed in Section 3 that a representation of the form $T^{i j}=T_{\mu}{ }^{i j} j_{\mu}$, with $T_{\mu}^{\prime}{ }^{i j} \in L_{10 c}^{\infty}(X, \mu)$ exists for every tensor measure. Suppose that holds; take $\psi_{i}=\xi_{i} \sqrt{\theta}$, with $\theta \in D^{\circ}\left(X, \mathbb{R}^{+}\right)$and $\xi=\left(\xi_{1}, \ldots, \xi_{n}\right) \in \mathbb{R}^{n^{n}} ;$ then

$$
\int \theta \xi_{i} \xi_{j} T_{\mu}{ }^{i} \mathrm{j} d \mu \geqslant 0
$$

This shows that $\xi_{i} \xi_{j} T_{\mu}^{, i j}(x) \geqslant 0$ for every $x \in X$ with the possible exception of a $\mu$-negligible subset $N(\xi)$. If $\xi$ ranges over a countable dense subset $\Xi$ of $\mathbb{R}^{n}$, the union of $N(\xi), \xi \in \Xi$, is $\mu$-negligible. Hence, for $\mu$-almost every $x$ in $X$, the quadratic form $\xi+\xi_{i} \xi_{j} T_{\mu}{ }^{i j}(x)$ in nonnegative on $\Xi$; by density it is nonnegative on the whole of $\mathbb{R}^{n}$.

REMARK.l. We have defined in the foregoing some infinite-dimensional manifold $M$ as the totality of the doubly contravariant tensir measures in $X$ which result from one of them through the transport by carriers with $D^{1}$ velocity fields. Clearly properties (a) and (b) are possessed by every element of $M$ as soon as this is true for one of them. Such is the case for the tensor measure considered in Section 6 , namely $T^{i j}=u^{i} j_{\mu}$, with $\mu$ a nonnegative real measure.

REMARK 2. We restricted ourselves in the preceding to the transport of tensor measures. Actually, supposing again for simplicity that the manifold $X$ is locally Euclidean, one may take as $T^{i j}$ the components of a symmetric tensor distribution of order $m$. If the velocity field of a carrier $\Lambda$ belongs to $D^{m+1}\left(X, X^{\prime}\right)$, the transport of the tensor distribution $T$ makes sense, generating 
a moving tensor distribution $T^{T}$ in $X$. Suppose, on the other hand, the real expression $J=\left\langle g_{i j}, T^{i j} \gg\right.$ meaningful; this is true in particular if $T$ has compact support. Thereby a real function $\tau \rightarrow J(\tau)$ is defined for $\tau$ in a neighborhood of zero. The derivative $j$ of this function may be calculated as in Section 5 ; again it turns out that $j(0)$ vanishes iff $T^{i j}, j=0$. When this holds, one may come to the calculation of the second derivative; like in Proposition 7.1 one finds

$$
\ddot{\mathrm{J}}(0)=2 \ll \mathrm{g}_{\mathrm{ij}} \varphi^{\mathrm{i}}, \mathrm{k} \varphi^{\mathrm{j}}, \ell, \mathrm{T}^{\mathrm{k} \ell} \gg,
$$

a meaningful expression since $\varphi \in D^{\mathrm{m}+1}\left(X, X^{\prime}\right)$. Similarly to Proposition 7.2 , this expression is found nonnegative if $f$, for every $\psi \in D^{\infty}\left(X, X^{\prime}\right)$, one has $\ll \psi_{i} \psi_{j}, T^{i j} \gg \geqslant 0$. Now this property implies that the tensor distribution $\mathrm{T}$ is actually a measure. The proof [8] is easily based on the classical fact that any nonnegative real distribution equals a measure.

In the applications of the transport method, the nonnegativity of $\ddot{\mathrm{J}}(0)$ appears as a natural "stability" requirement. For this reason the presentation of the method may practically be restricted to the case of tensor measures.

\section{CONCLUSION}

The logical pattern of the foregoing may be summarized as follows.

In a reference manifold $X$ (this equals in practical instances an open subset of Euclidean $\mathbb{R}^{n}$ ) some investigated object $U$ is required to satisfy a certain system of partial differential equations. Nonsmooth solutions are expected, i.e. the unknown object $U$ is an element of some space $J$ of distributions with prescribed tensorial type and distributional order.

Here are the essentials of the transport method.

A reference field $r$ is specified in $X$. The preceding sections have been restricted to the case where $r$ equals the doubly covariant tensor field $g$ defining in $X$ some Riemannian 
metric (and for simplicity most calculations have been performed with a Euclidean metric). Generally $r$ may be a field whose tensoriel type and order of differentiability match the tensorial type and distributional order of $U$; in other words, for every $U$ in $J$, the real valued linear functional $U \rightarrow \ll r, U \gg$ makes sense.

A carrier $\Lambda$ is defined in $X$ by its velocity field, say $\varphi \in D^{k}\left(X, X^{\prime}\right)$, with $k$ large enough for the transport of elements of $J$ to be defined. For instance, $k=1$ allows for the transport of tensor measures ; generally the transport of tensorial objects with distributional order $\mathrm{k}-\mathrm{l}$ is meaningful. As indicated in Section 4, $\varphi$ may also depend on the formal time $\tau \in I$ ( $I$ is an open real interval containing zero) with continuous differentiability up to order $k$ in $I \times X$; in that case it is assumed that the support of $x+\varphi(\tau, x)$ is contained in a. $\tau$-constant corpact subset of $x$.

Since $\varphi$ belongs to $D^{k}$, the carrier $\Lambda$ may be endowed with the structure of a $c^{k}$-manifold, in such a way that, for every $\tau \in I$, the placement mapping $p_{\tau}: \Lambda \rightarrow X$ is a $c^{k}$ diffeomorphism. Assume that the tensor field $r$ is $c^{k-1}$ in $X$; then its image under $\mathrm{p}^{-1}$ is a $c^{k-1}$ tensor field in $\Lambda$, say $\rho^{\tau}$. For every $\lambda \in \Lambda$, the value $\rho^{\tau}(\lambda)$ constitutes a $\tau$-depending element of the tensorial product of some copies of the tangent and/or cotangent space to $\Lambda$ at point $\lambda$. The smoothness assumptions made imply that the $\tau$-derivative $\dot{\rho}^{\top}(\lambda)$ exists and that $\lambda \rightarrow \dot{\rho}^{\top}(\lambda)$ is a $c^{k-1}$ field on $\Lambda$, of the same tensorial type as $\rho^{\top}$, with compact support. Taking, for $\tau=0$, the image of $\dot{p}^{\top}$ under $p$ yields a $c^{k-1}$ tensor field on $x$, with compact support; this is, by definition, the Lie derivative $\mathrm{L}_{\varphi} \mathrm{T}$.

The reference. field $r$ has been assumed to belong to $c^{k}(x, T)$, where $T$ denotes a certain tensorial type, possibly astrained to some conditions of symmetry or skew-symmetry. Therefore, $\varphi \rightarrow \mathrm{L}_{\varphi} \mathrm{r}$ is a continuaus linear mapping, say $\mathrm{D}$, of $D^{k}\left(X, X^{\prime}\right)$ to $D^{k-1}(X, 7)$. In the preceding sections, restricted to the case $r=g, D$ equalled twice the operator def ; the divergence operator, acting on symmetric tensor measures, emerged as the negative transpose of def. Generally, the transpose of $D$ is a linear opera- 
tor acting on distributions, say $D^{*}: D^{k^{-1}}\left(X, T^{*}\right) \rightarrow D^{k}\left(X, X^{*}\right)$; here $T^{*}$ refers to the tensorial type dual of $T$. The transport method provides a variational characterization of the solutions $U$ to $D^{*} U=0$, or more generally to $\mathrm{U}^{*} \mathrm{U}=\mathrm{f}$, provided the righthand nember $f$ has an adequate form.

For more comments on the prospect of this method, let us again restrict ourselves to the special case $r=g$, with $X$ equal to an open subset of a Euclidean space. Using orthonormal Cartesian coordinates in this space, one looks for a symmetric tensor measure $\mathrm{T}^{\mathrm{ij}}$ with zero divergence, i.e. $\mathrm{T}^{\mathrm{i} j}, \mathrm{j}=0$ in $X$. We have interpreted Proposition 5.1 by saying that $T$ verifies this equation iff it constitutes a critical point of the real functional $J$ on some infinite-dimensional manifold $M$. This manifold consists of the tensor measures obtained by transporting $T$ along carriers with velocity fields in $D^{l}\left(X, X^{\prime}\right)$. The computational use of this remark may be contemplated by discretizing the carrier as a moving finite element mesh. The approximate representation of a tensor measure relatively to such a mesh, and of its transport when the mesh deforms, are easily imagined. Starting with a chosen tensor measure $T_{0}$, the mesh will be deformed stepwise. If $T_{0}$ and therefore all the tensor measures obtained by transporting it possess the nonnegativity property involved in Proposition 7.2 , each step of mesh displacement will be devised so as to generate a walk toward a minimal point of $\mathrm{J}$.

The convergence of the process is naturally related to the boundary conditions one intends to satisfy. All the preceding has been developed with a carrier $\Lambda$ whose velocity field has compact support in $X$. The mechanical analog is a continuous medium whose boundary particles are fixed. Determining a placement $p: \Lambda \rightarrow X$ which minimizes the real functional $J$ under such a boundary constraint may then be seen as a special problem of hyperelastic equilibrium. With the notations of Sections 4 and 5 , the corresponding elastic energy is expressed as $\int_{\Lambda} \gamma_{i j} d \theta^{i j}$; here recall that $\theta$ is a doubly contravariant tensor measure, constant on the manifold $\Lambda$. The doubly covariant tensor field $\gamma$, i.e. the image of $g$ under $p^{-1}$, depends quadratically on the tangent mapping $\partial p / \partial \lambda$; 
in fact, if $p^{k}\left(\lambda^{l}, \ldots, \lambda^{n}\right)$ denote the components of $p(\lambda)$ relative to some orthonormal Cartesian frame of $x$, one has

$$
\gamma_{i j}(\lambda)=\frac{\partial p^{k}}{\partial \lambda^{i}} \frac{\partial p^{k}}{\partial \lambda^{j}} \text {. }
$$

This makes the determination of the equilibrium placement $p$ a boundary value problem concerning a linear system of partial differential equations in the $\lambda^{i}$ variables, with a priori nonsmooth coefficients. of course, the existence of solutions can on $1 y$ be expected in a weak sense, involving placements which are no more $c^{l}$ mappings of $A$ onto $X$ but only elements of some sobolev spaces. The numerical treatment of this system of partial differential equations requires a mesh in the manifold $\Lambda$ : this is the same as the moving mesh considered in the preceding.

This throws some light on the structure of the set of the solutions to the equations of Hydrodynamics, as investigated in Section 6. These equations do no actually reduce to the simple form $\mathrm{T}^{\mathrm{ij}}, \mathrm{j}=0$, but the analogy with a problem of hyperelastic equilibrium is not destroyed by the additional terms; the gravity term $\rho U, i^{\ell}$ in (6.1) only plays the part of a loading (a "dead loading" in the usual case of constant gravity) while the pressure term modifies the density of elastic energy by adding a function of the Jacobian determinant of the placement. The essential nonlinearity of the equations of Hydrodynamics lies in the algebraic structure of the tensor measure $\mathrm{T}^{\mathrm{ij}}$ : its density, relative to the scalar measure $\rho \ell$, equals the tensor product of the vector field $u$ by 1 tself. Provided that the approximation process is initiated with a tentative solution $T_{0}$ which meets such a requirement, the transpirt method handles this condition automatically; the nonnegativity property of Proposition 7.2 is also secured by itself. Observe that, if the hyperelastic analogy is brought about in numerical prosedures, the constraint imposed to the fictitious elastic medium $\Lambda$ may be relaxed : instead of assuming each boundary particle fixed, one may permit the medium to slide along some part $S$ of the boundary of the region $X$. If the approximation process is initiated with a fluid velocity field $u_{o}$ tangential to $s$ - a usual circumstance in hydrodynamical problems-the transport by $\Lambda$ will preserve this condition. 


\section{REFERENCES}

[1] P.R. Garabedian and D.C. Spencer. Extremal methods in cavitational flow, J. Rational Mech. and Anal., I(1952), 359-409.

[2] P. Casal. Sur l'énergie cinétique d'un écoulement possédant une surface de discontinuité de vitesse, C.R. Acad. Sci. Paris, 234 (1952), 804-806.

[3] J.J. Moreau. Fluid dynamics and the calculus of horizontal variations, Int. J. Engng. Sci. 20(1982), 389-411.

[4] J.J. Moreau. Variational properties of stationary inviscid incompressible flows with possible abrupt inhomogeneity or free surface, Int. 3. Engng. Sci. 23(1985), 461-481.

[5] N. Bourbaki. Integration, Hermann, Paris.

[6] J.J. Moreau. Le transport d'une mesure vectorielle par un fluide et le théorème de Kelvin-Helmholtz, Rev. Roum. Math. Pures et Appl. 27(1982), 375-383.

[7] J.E. Marsden and T.J.R. Hughes. Mathematical foundations of elasticity, Prentice-Ha11, 1983.

[8] M. Ros. Formes quadratiques positives sur des espaces de gradients, Séminaire d'Analyse Convexe, USTL, Montpellier, 7 (1977), exp. $n^{\circ} 5$.

[9] G. De Rham. Variétés différentiables, Hermann, 1955. 